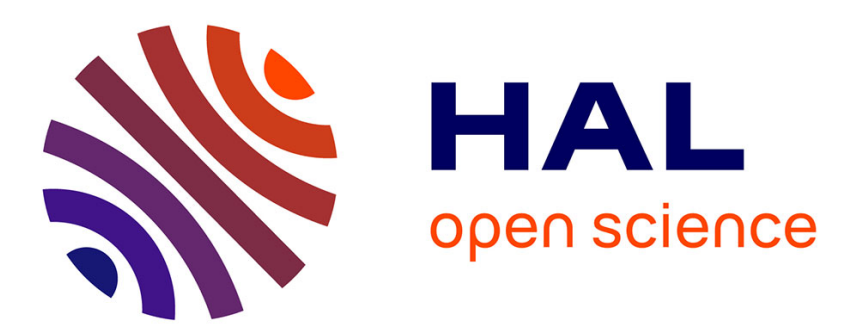

\title{
Extreme scenarios for the evolution of a soft bed interacting with a fluid using the Value at Risk of the bed characteristics
}

Bijan Mohammadi, Frédéric Bouchette

\section{- To cite this version:}

Bijan Mohammadi, Frédéric Bouchette. Extreme scenarios for the evolution of a soft bed interacting with a fluid using the Value at Risk of the bed characteristics. Computers and Fluids, 2014, 89, pp.78-87. 10.1016/j.compfluid.2013.10.021 . hal-01054937

\section{HAL Id: hal-01054937 https://hal.science/hal-01054937}

Submitted on 10 Aug 2014

HAL is a multi-disciplinary open access archive for the deposit and dissemination of scientific research documents, whether they are published or not. The documents may come from teaching and research institutions in France or abroad, or from public or private research centers.
L'archive ouverte pluridisciplinaire HAL, est destinée au dépôt et à la diffusion de documents scientifiques de niveau recherche, publiés ou non, émanant des établissements d'enseignement et de recherche français ou étrangers, des laboratoires publics ou privés. 


\title{
EXTREME SCENARIOS FOR THE EVOLUTION OF A SOFT BED INTERACTING WITH A FLUID USING THE VALUE AT RISK OF THE BED CHARACTERISTICS
}

\author{
COMPUTERS \& FLUIDS, 89 (2014). \\ BIJAN MOHAMMADI \\ INSTITUT DE MATHÉMATIQUES ET DE MODÉLISATION DE \\ MONTPELLIER \\ UNIVERSITÉ MONTPELLIER II \\ CC51, 34095 MONTPELLIER, FRANCE \\ FREDERIC BOUCHETTE \\ GÉOSCIENCES MONTPELLIER \\ UNIVERSITÉ MONTPELLIER II \\ CC60, 34095 MONTPELLIER, FRANCE
}

\begin{abstract}
We show how to introduce the Value at Risk (VaR) concept in the analysis of the adaptation of the shape of a soft bed to a flow knowing the Probability Density Function (PDF) of the responses of the shape to flow perturbations (bed receptivity). Our aim is to quantify our confidence on simulation scenarios by an available morphodynamic model for the shape. The approach permits to perform this task at low complexity as it does not require any sampling of the bed receptivity parameter space. The paper goes beyond stationarity for the variability of the bed receptivity by linking its dynamics to the bottom morphodynamics through an original transport equation for the local bed receptivity standard deviation. The approach has been applied to the analysis of bed morphodynamics based on minimization principles. The results show the importance of including uncertainty information during the coupling and not only eventually through simple margins on the results.
\end{abstract}

Key words and phrases. Value at Risk, uncertainty, shape deformation, shape optimization, beach morphodynamics, Saint Venant Equations, sensitivity analysis, worst-case analysis. 


\section{INTRODUCTION}

There is an increasing demand to account for data uncertainties in simulation and design. We are interested by situations where a PDE based state equation is involved as typically in a flow problem in a bounded domain. An important situation is where the boundary of the domain, or some part of it (e.g. a shape in a flow), evolves under the action of the flow. Examples of such interactions can be seen in the thermal ablation of a shape or in the changes of a sandy bed of a river or sea. Obviously, these boundary evolutions involve different sorts of materials, flow constituants and physics but share a same global philosophy: they permit to dump or dissipate some energy expression.

Consider the following simulation chain linking a control parameter $\rho$, its random perturbation $\varepsilon(\rho)$ known through a probability density function (PDF) $(\tilde{\rho}$ being then the perturbed control parameter), a state variable $u(\tilde{\rho})$ and a cost function $J$ :

$$
\rho \rightarrow \tilde{\rho}=\rho+\varepsilon(\rho) \rightarrow u(\tilde{\rho}) \rightarrow J(u(\tilde{\rho}))
$$

Without being exhaustive, to handle these perturbations one classical approach is to propagate the randomness into the whole chain and get a noisy functional. And then assimilate, in a least-square sense for instance, this functional by a lowcomplexity model (e.g. a parametric representation of the functional) and later use this reduced-order model instead of the original one. This approach is widely used in robust engineering optimization and demands for a priori regularity hypothesis for the reduced-order model and trust regions definition. Another possibility is to define the momentum of our noisy functional in momenta-based optimization (e.g. minimizing the mean while keeping the variance unchanged). This is also very much used in practice if the number of control parameters is small and the complexity of each simulation reasonable. These approaches receive different denominations following the field of research they are issued from: learning in neural networks, fitting with least squares in experimental plans or higher degrees polynomials, reduced-order 
modeling with proper orthogonal decomposition or other low-complexity modeling $[1,2,3,4,5,27,26]$.

Our motivation is somehow different. Indeed, relation (1) represents an important situation in which where randomness appears is well identified. Indeed, if $\varepsilon(\rho)=0$ the simulation is fully deterministic. We would like to keep it so and still take into account these random perturbations through extreme scenarios derived by the introduction of a priori information on $\varepsilon(\rho)$ into the simulation chain. And, to keep the complexity low, this should be achieved without any sampling of the control space.

Extreme scenarios are interesting in environmental simulations as they provide an easy tool for risk quantification. One application of the present contribution is to permit the derivation of extreme scenarios for the evolution of the shape of soft beds following previous contributions on the application of control theory to the evolution of sandy sea beds $[29,30]$ which are useful, for instance, when designing defense structures against beach erosion $[9,10,11]$. We model sea bed evolution through fluid and structure coupling. The fundamental assumption in this interaction is that the bed adapts to the flow by some sort of optimal sand transport in order to minimize the flow energy or some other functional. The literature on coastal morphodynamics is huge $[12,13,14,15,16]$ and concerns phenomena with very different time scales, as for instance, sediment transports using fluid-induced shear in the sediment modelling, sea bed friction and the feedback of bed shapes on the flow, dunes morphodynamics, global beach morphodynamics based on long and crossshore fluxes and beach lines morphodynamics. In our model, the time scales of interest are rather small and recoveries between storms or seasonal and inter-annual variabilities are out of scope.

Several quantities can be considered as uncertain in the bed definition (e.g. bed receptivity to flow perturbation) as well as in the flow characteristics (e.g. wave amplitudes, frequencies and directions). In this work we consider variabilities on the bed receptivity $(\rho \geqslant 0)$ we would like to account for in the evolution of the bed 
through two extreme scenarios. Each scenario is based on a local threshold value for the bed receptivity defined for a given probability level through $\operatorname{VaR}_{\alpha \in[0,1[}^{ \pm}$. Hence, we can give information such as: following our knowledge of the uncertainties on the bed (PDF) and based on the physics encapsulated in the models, there is $\alpha \times 100 \%$ chances for the evolution of the bed to take place between these two scenarios. We consider Gaussian PDF, but the approach is not limited to those. Gaussian PDFs are interesting as they permit to easily express the VaR from the standard deviation. Usually the variability on an uncertain parameter is assumed stationary. One originality in this paper is the introduction of a transport equation for the standard deviation following the evolution of the bed. The stationary variance case can then be treated as a particular situation.

The paper starts with the concept of the Value at Risk. Then a model problem is proposed to illustrate the different ingredients in the coupling between a soft shape and a flow based on the minimization of some energy quantity. The variability of the shape characteristics is then accounted for in the minimization and it is shown how to introduce an equation to model the dynamics of this variability following the shape deformation. The approach is made more realistic with the introduction of the Saint Venant flow model for shallow waters. The paper also shows several simulations with quantitative extreme scenarios. The simulations show the importance of including VaR information all along the coupling and not only eventually through security margins as often in engineering approaches. Indeed, we see that the deviations between the simulations are not necessarily correlated with the uncertainty distributions.

\section{Value at Risk (VAR)}

In financial engineering, the Value at Risk (VaR) is a widely used risk measure of the risk of loss on a given asset [25]. It defines, for a given probability level $(0<\alpha<1)$ and time horizon (typically one day), a threshold value for the loss $X$ 
on the asset:

$$
\operatorname{VaR}_{\alpha}=\inf \{l \in \mathbb{R}: P(X>l) \leqslant 1-\alpha\} .
$$

This is illustrated in figure 1 where the areas filled worth $\alpha$. We would like to use this concept during our simulation knowing the PDF of the uncertainties on the bed receptivity. Hence, during our fluid-structure coupling the bed receptivity $\rho$ represents, with a confidence level of $\alpha$, the interval $\left[\max \left(0, \rho+\operatorname{VaR}_{\alpha}^{-}\right), \rho+\operatorname{VaR}_{\alpha}^{+}\right]$ around $\rho$ the value one would have used for the bed receptivity in a deterministic simulation and $\mathrm{VaR}_{\alpha}^{-} \leqslant 0 \leqslant \mathrm{VaR}_{\alpha}^{+}$. One expresses then possible deviations from $\rho$ via two PDF and the corresponding values at risk. One can make the hypothesis that the upper and lower bounds of the variations are symmetric, in which case $\operatorname{VaR}_{\alpha}^{-}=-\mathrm{VaR}_{\alpha}^{+}$. This is typically the case when uncertainties on a parameter follow a Gaussian distribution. We will introduce a dynamic model for the variability to address the time issue and the fact that the VaR may also be time dependent. This makes sense as, indeed, when the bed changes its local receptivity might change as well. In this case the stationary situation is treated as a particular case.

In the context of Gaussian probability density functions values at risk are explicitly known: $\operatorname{VaR}_{0.99}=2.33$ and $\operatorname{VaR}_{0.95}=1.65$ for $N(0,1)$ and $\operatorname{VaR}_{\alpha}(N(0, \sigma))=$ $\sigma \operatorname{VaR}_{\alpha}(N(0,1))$. We suppose Gaussian PDF for the bed receptivity $\rho$ and its VaR is therefore known.

The choice for a positive $\rho$ has two reasons. $\rho$ should be linked with the bed porosity $(\eta \geqslant 0)$ in bed transport models such as equation (17) and we would like to use it in a minimization procedure. In other words, we make the assumption that the bed will take the steps which will decrease the functional (see sections 3 and 4).

\section{A Simple illustrative model}

Let us illustrate our purpose on a model problem based on an analytical expression for the variations of a surface $h$. Consider a domain with a bed defined as a function $\psi(t, x): \mathbb{R}^{+} \times[-100 m,-20 m] \rightarrow \mathbb{R}^{+}$. We want to model the changes in this bed with time due to elevations $h(t, x)$. With the notations of the chain $(1) u=(\psi, h)$. 
Let us start with the following simple expression for $h$ :

$$
h(t, x)=h_{0}+\beta \sin \left(\omega x\left(h_{0}-\psi(t, x)\right)\right),
$$

where $h_{0}$ indicates the level at rest, $\beta$ and $\omega$ are given such that $0 \leqslant \beta<1$ and $\omega>0$. This is a monochromatic wave, but one could consider superimposed waves as in section 6 . We consider $h_{0}=1.3 \mathrm{~m}, \omega=1 H z$ and $\beta=0.1 \mathrm{~m}$. The bed is supposed initially flat $\psi(0, x)=1 \mathrm{~m}$. To close this model, we need to provide a model for $\psi(t, x)$ and $\rho(t, x)$. We suppose that $\psi$ will adapt to the flow elevation in order to reduce a functional $J(h)$ given by the $L^{2}$ norm of $h$ in space:

$$
J(h)=\frac{1}{2 h_{0}^{2}} \int_{-100}^{-20} h^{2}(t, x) d x .
$$

The scaling coefficient is chosen in order for $\nabla_{\psi} J$ to be dimensionless. The choice of the functional is not central in our discussion.

An example of evolution model from time $t$ to time $t+\delta t$ for this system can be:

$$
\begin{gathered}
\psi(t+\delta t, x)=\psi(t, x)-\delta t \rho(t, x) \nabla_{\psi} J(\psi(t, x), h(t, x)), \\
h(t, x)=h_{0}+\beta \sin \left(\omega x\left(h_{0}-\psi(t+\delta t, x)\right)\right) .
\end{gathered}
$$

$\rho(t, x)(m / s) \geqslant 0$ models the receptivity of the bed and depends on both time and space as the bed is usually non uniform. If $\rho$ is assumed stationary in time one simply has $\rho(t, x)=\rho_{0}(x)$ given. Variability on the bed receptivity can be introduced and accounted for during the simulation through VaR information. For instance, if we assume Gaussian PDF for the bed receptivity with its standard deviation $\sigma(x)$ known and $\operatorname{VaR}_{0.95}^{ \pm}(N(0, \sigma(x)))= \pm 1.65 \sigma(x)$. Two scenarios can be considered therefore with $\rho_{0}(x)+\operatorname{VaR}_{0.95}^{ \pm}(N(0, \sigma(x)))$.

One way to go beyond the stationarity hypothesis for $\rho$ is to consider $\sigma$ being also a function in time and introduce an extra transport equation for $\sigma(t, x)$. This is, for instance, useful if the variability changes with the motion of the bed. Let us rewrite 
the evolution for $\psi$ above as governed by a transport equation:

$$
\begin{aligned}
\psi(t+\delta t, x) & =\psi(t, x)-\delta t \rho(t, x) \nabla_{\psi} J(\psi(t, x), h(t, x)) \\
& =\psi(t, x)-\delta t V(t, x) \partial_{x} \psi(t, x)
\end{aligned}
$$

Eq. (4) permits to define $V(t, x)=\rho(t, x) \nabla_{\psi} J / \partial_{x} \psi$ as the 'equivalent' local speed of the motion of the bed (see figure 2 for snapshots of $V$ ).

Now the evolution of local variability can be linked to $V$ by:

$$
\sigma(t+\delta t, x)=\sigma(t, x)-\delta t V(t, x) \partial_{x} \sigma, \sigma(t=0, x)=\sigma_{0}(x)
$$

This transport equation is completed by characteritics boundary conditions following the sign of $V$ at each of the boundaries.

This model suggests that the bed velocity increases when the bed is or gets flat. In other words, flat beds appear being unstable which is something one observes in nature. Another way to write the dynamic of $\sigma$ is by the introduction of variable time scales for the bed following its slope (see also section 4):

$$
\sigma(t+\delta t, x)=\sigma(t, x)-\tilde{\delta} t \rho(t, x) \nabla_{\psi} J \partial_{x} \sigma, \sigma(t=0, x)=\sigma_{0}(x),
$$

with $\tilde{\delta t}=\delta t / \partial_{x} \psi \rightarrow \infty$ when the bed gets flat which suggests one should look for the steady solution of (6) in such regions where iso- $\sigma$ appears parallel to the variation of the shape. In realistic situations, one should not dissociate the sensitivity of the functional with respect to the bed $\nabla_{\psi} J$ from the local bed slope $\partial_{x} \psi$. Indeed, we will see in section 4 with the Saint Venant shallow water equations [6] as state equations that the bed slope is actually accounted for in the state equations and therefore in their linearization leading to $\nabla_{\psi} J$.

Now let us account for this variability in a numerical simulation. We assume Gaussian PDF for the bed receptivity with its initial standard deviation increasing linearly through the domain and $\operatorname{VaR}_{0.95}^{ \pm}(N(0, \sigma))= \pm 1.65 \times 10^{-3}\left|\frac{x+100}{100}\right|$ as shown in figure 2. The figure also shows the evolutions of the bottom shapes and water elevations with 70 iterations of (3) with $\delta t=2 s$ on a 500 points mesh when the bed 
receptivity follows the two extreme scenarios mentioned. The evolution of the bed receptivity variability following (5) is also shown in the picture. One can see that in both extreme cases the bottom has adapted in order to reduce surface elevations. The deviation between the two solutions increases with the variability. Obviously, the elevation reduction is less efficient when the bed receptivity is lower. One will see in section 6 that these informations are not necessary correlated. This approach permits therefore to link data variability to the solution of a model and exhibit solution ranges with a quantifiable statistical theoretical background.

\section{INTERACTIONS BETWEEN THE FLOW AND ITS BED}

Now, we consider two dimensional situations with a realistic flow model in shallow domains. The sea bed $\psi$ changes with time following the changes in the state given by the flow variables $\mathbf{U}$. We use a bed parameterization based on the bathymetry given at all the nodes of the fluid mesh.

The model for the sea bed evolution is based on the minimization of a time dependent functional $J\left(\psi,\left\{\mathbf{U}(\psi(\tau, x), \tau, x), \tau \in[t-T, t], x=\left(x_{1}, x_{2}\right) \in \Omega \subset \mathbb{R}^{2}\right\}\right)$ involving the state evolution in time, solution of a state equation.

We consider the Saint Venant shallow water equations as state equations for the fluid with $\mathbf{U}={ }^{t}(h, h \mathbf{u})$ where $\mathbf{u}={ }^{t}(u, v)$ is the depth-averaged velocity with $u$ and $v$ the scalar components in the horizontal directions and $h$ the local water depth:

$$
\partial_{t} \mathbf{U}+F(\mathbf{U}, \psi)=0 \text {, with initial and boundary conditions, }
$$

where

$$
F(\mathbf{U}, \psi)=\left(\begin{array}{c}
\nabla \cdot(h \mathbf{u}) \\
\nabla \cdot(h \mathbf{u} \otimes \mathbf{u})+g h \nabla(h+\psi)
\end{array}\right)
$$

where $g\left(m . s^{-2}\right.$ is acceleration due to gravity. 
As in our illustrative problem of section $3, J$ is minimized looking for the evolution in time of $\psi$ following:

$$
\partial_{t} \psi=-\rho(t, x) \nabla_{\psi} J, \psi(t=0, x)=\psi_{0}(x)=\text { given },
$$

where $\rho(t, x)$ is a positive parameter which depends on the local porosity of the bed and quantify its receptivity to flow perturbations. This can also be linked to transport models for the bed such as the Exner equation described in section 4.4. The physical time scales for the fluid and structure (bed motion) are quite different. Indeed, flows have time scales of the order of seconds and the sea bed motion takes place over hours as we will see in section 6 .

4.1. Cost function . The cost function involves the state evolution as, for instance, in:

$$
J(\psi)=K \int_{t-T}^{t} j(\psi, \mathbf{U}(\psi, \tau)) d \tau,
$$

where $T$ indicates a time dependency window and also permits to introduce a difference in time scales between sea bed and flow motions. The scaling $K$ is to make $\nabla_{\psi} J$ dimensionless. One looks for the bed acting as a flexible structure and adapting to the flow conditions in order to minimize some energy-based functional. In $[29,30]$ one considers a functional made of the $L^{2}$ norm of the deviation of the wave elevation from a low frequency component evaluated using a time interval $T$ : $\eta(x, t)=h(x, t)-\frac{1}{T} \int_{t-T}^{t} h(x, \tau) d \tau$ and involving a constraint on sand displacements requiring minimal topobathymetry changes from the bathymetry $\psi(t-T)$ at the beginning of a time interval of influence $[t-T, t]$ :

$$
J(\psi)=\frac{1}{|\Omega| T h_{0}} \int_{t-T}^{t} \int_{\Omega}\left(\eta^{2}+(\psi(\tau)-\psi(t-T))^{2}\right) d \tau d \Omega
$$

where $\Omega$ is the observation domain and $h_{0}$ is the water level at rest. Alternatively, in this work we give examples of simulations (see section 6) with another functional which aims at minimizing water elevations and their spatial gradients on a given 
time interval $T$ :

$$
J(\psi)=\frac{1}{|\Omega| T} \int_{t-T}^{t} \int_{\Omega}|\eta|\left\|\nabla_{x} \eta\right\| d \tau d \Omega .
$$

4.2. Variability dynamics. Proceeding as in our model problem, we introduce the bed speed and rewrite

$$
\rho \nabla_{\psi} J=V(t, x) \nabla_{x} \psi(t, x)=v_{1} \partial_{x_{1}} \psi+v_{2} \partial_{x_{2}} \psi
$$

which permits, after multiplying by $\partial_{x_{1}} \psi \partial_{x_{2}} \psi$, to introduce a transport equation for $\sigma(t, x)$ characterizing the dynamics of the variability of $\rho(t, x)$ :

$$
\left|\partial_{x_{1}} \psi \partial_{x_{2}} \psi\right| \partial_{t} \sigma+S \rho \nabla_{\psi} J\left(\partial_{x_{2}} \psi \partial_{x_{1}} \sigma+\partial_{x_{1}} \psi \partial_{x_{2}} \sigma\right)=0
$$

with an initial condition $\sigma(t=0, x)=\sigma_{0}(x)$ and $S=\operatorname{sgn}\left(\partial_{x_{1}} \psi \partial_{x_{2}} \psi\right)$. This transport equation is completed by either symmetry or characteristic boundary conditions (denote $n=\left(n_{1}, n_{2}\right)$ the local outward normal to the boundary):

$$
\nabla_{x} \sigma . n=0 \text { or } \sigma(t, x)=\text { given if } S \rho \nabla_{\psi} J\left(\partial_{x_{2}} \psi n_{1}+\partial_{x_{1}} \psi n_{2}\right)<0
$$

This model suggests that the time scale is locally modified following the local geometry of the bed expressed through $\left|\partial_{x_{1}} \psi \partial_{x_{2}} \psi\right|$. For instance if this quantity vanishes (i.e. the bed is flat in some direction) one should consider stationary solution of (13) (see figure 3 for an example). This is therefore a very stiff equation to solve. Figure 4 shows an example of $\rho_{0}(x)+\sigma_{0}(x)$ and $\rho(t, x)+\sigma(t, x)$.

4.3. Conservation . If the simulation takes place in a closed domain $\Omega^{\prime}$ the amount of the material making the bed must be conserved:

$$
\int_{\Omega^{\prime}} \partial_{t} \psi d \omega=0 \text { or } \int_{\Omega^{\prime}} \psi d \omega=C=\text { constant. }
$$

In conservation form the analysis above makes looking for $V$ such that:

$$
\rho \nabla_{\psi} J=\nabla_{x} \cdot(\psi(t, x) V(t, x))=\partial_{x_{1}}\left(\psi v_{1}\right)+\partial_{x_{2}}\left(\psi v_{2}\right)
$$


which gives in integral form a constraint on the field $V$ :

$$
\begin{aligned}
\int_{\Omega^{\prime}} \nabla_{x} & \cdot(\psi V) d \omega=\int_{\partial \Omega^{\prime}} \psi(V . n) d \gamma \\
= & \int_{\Omega^{\prime}} \rho \nabla_{\psi} J d \omega=0 .
\end{aligned}
$$

Expressing $V=\left(\rho \nabla_{\psi} J / \partial_{x_{1}} \psi, \rho \nabla_{\psi} J / \partial_{x_{2}} \psi\right)$ and $n=\left(n_{1}, n_{2}\right)$ one has:

$$
\begin{gathered}
\int_{\Omega^{\prime}} \partial_{x_{1}}\left(\frac{\psi \rho \nabla_{\psi} J}{\partial_{x_{1}} \psi}\right)+\partial_{x_{2}}\left(\frac{\psi \rho \nabla_{\psi} J}{\partial_{x_{2}} \psi}\right) d \omega=\int_{\partial \Omega^{\prime}} \psi \rho \nabla_{\psi} J\left(\frac{n_{1}}{\partial_{x_{1}} \psi}+\frac{n_{2}}{\partial_{x_{2}} \psi}\right) d \gamma \\
=\int_{\Omega^{\prime}} \rho \nabla_{\psi} J d \omega=0
\end{gathered}
$$

For $\nabla_{\psi} J$ to be compatible with this constraint on $V$, the cost function (9) must be augmented by a penalty term (e.g. $\left.\left|\int_{\Omega^{\prime}} \psi d \omega-C\right| /\left|\Omega^{\prime}\right|\right)$ enforcing constraint (14).

Let us analyze expression (16) supposing $\psi>0$ and $\rho>0$. The condition on $\rho$ means that the bed is receptive to perturbations everywhere and has no solid parts $(\rho=0)$. In all cases, it is difficult in practice to enforce a no bed motion just along the boundaries in an experiment with a sandy bed. One way to be sure for the last expression to vanish for all cost functions $J$ is to have $n_{1} \partial_{x_{2}} \psi=-n_{2} \partial_{x_{1}} \psi$ which is difficult to realize. Indeed, consider $\Omega^{\prime}$ to be square as in figure 4 . The unit normals along the boundaries of this domain are either $( \pm 1,0)$ or $(0, \pm 1)$. The only shape for which this condition is surely realized is a flat bed $(\psi=c s t e)$ which we saw is an unstable shape. So the best way to make sure that the conservation constraint on $\mathrm{V}$ is realized is for $\nabla_{\psi} J$ to vanish along the boundaries. This means that the observation domain $\Omega$ used in the definition of the cost function should be small compared to the domain $\Omega^{\prime}$. However, for experiences taking place over a long period of time, it is impossible to remove the influences of the boundaries of $\Omega^{\prime}$ on what is happening in $\Omega$. This brings therefore the question of the pertinence of extending conclusions on a bed long-term evolution obtained from an experiment in a basin to open seas (see section 6 for an example). 
4.4. Application with the Exner equation. The present analysis of extreme scenarios using the VaR concept can be applied to other models of bed motion available in the literature as, for instance, the Exner equation [20] which models the conservation of mass between 'in the bed' and 'transported' sediments:

$$
\psi_{t}=-\frac{1}{1-\lambda_{p}(t, x)} \nabla \cdot q, \quad \psi(t=0, x)=\text { given }
$$

where $\lambda_{p} \in[0,1[$ is the porosity of the bed or void fraction (i.e. a fraction of the volume of voids over the total volume). This coefficient plays the role of $\rho$ in the minimization equation (8): bed receptivity increases with $\lambda_{p} . q$ is a function of the flow variables. In $[29,30]$ one shows that equation (8) is a particular Exner equation with a non local flux similar to one in a model given by Fowler [22]. At this point, if the PDF of the variability in $\lambda_{p}$ is known, it can be accounted for, in the same way than for $\rho$, through its VaR. This permits using a solution solver for the Exner equation to generate extreme scenarios of bed evolution.

\section{Sensitivity EVAluation}

Sensitivity evaluation in large dimension needs an adjoint variable approach to make the cost of the evaluation independent from the size of the control space. In time dependent problems, this implies storage of all intermediate states which can be optimized by check-pointing technics $[8,31]$. In this section, we briefly describe the adjoint method with a time dependent state equation (the Saint Venant equations in our case).

Let us consider the following formal dependency chain:

$$
\psi \rightarrow\{\mathbf{U}(\psi, \tau), \tau \in[0, T]\} \rightarrow J(\psi, T)
$$

Here, $\psi$ is the independent variable and $\mathbf{U}$ and $J$ are the dependent ones. $\mathbf{U}(\psi, \tau)$ are solutions in time of

$$
\partial_{t} \mathbf{U}+F(\mathbf{U}, \psi)=0, \mathbf{U}(0)=\mathbf{U}_{0}(\psi)
$$


To be accurate, one should have also to consider the independent physical parameters such as those describing the waves. But, this would have introduced unnecessary complications into notations.

Now consider a functional involving an integral over time like those we are interested in:

$$
J(\psi, T)=\int_{(0, T)} j(\psi, \mathbf{U}(\psi, t))
$$

Linearizing $J$ one has:

$$
J_{\psi}(\psi, T)=\int_{(0, T)}\left(j_{\psi}+j_{\mathbf{U}} \mathbf{U}_{\psi}\right)
$$

In this expression only $\mathbf{U}_{\psi}$ is costly to get as it requires the linearization of the shallow water equations.

The linearized state equation:

$$
\partial_{t}\left(\mathbf{U}_{\psi}\right)+F_{\psi}(\mathbf{U}, \psi)+F_{\mathbf{U}}(\mathbf{U}, \psi) \mathbf{U}_{\psi}=0, \mathbf{U}_{\psi}(0)=\mathbf{U}_{0}^{\prime}(\psi),
$$

permits to write for all function $\mathbf{V}$ (where $\mathbf{V}$ has the same structure than $\mathbf{U}$ ):

$$
0=\int_{(0, T) \times \Omega}\left(\partial_{t}\left(\mathbf{U}_{\psi}\right)+F_{\psi}(\mathbf{U}, \psi)+F_{\mathbf{U}}(\mathbf{U}, \psi) \mathbf{U}_{\psi}\right) \mathbf{V}
$$

Introducing the adjoint operator $F_{\mathrm{U}}^{*}$, it gives:

$$
0=\int_{(0, T) \times \Omega}\left(-\partial_{t} \mathbf{V}+F_{\mathbf{U}}^{*}(\mathbf{U}, \psi) \mathbf{V}\right) \mathbf{U}_{\psi}+\int_{\Omega}\left[\mathbf{V} \mathbf{U}_{\psi}\right]_{0}^{T}+\int_{(0, T) \times \Omega} \mathbf{V} F_{\psi}(\mathbf{U}, \psi) .
$$

Let us introduce a backward adjoint problem:

$$
\partial_{t} \mathbf{V}+F_{\mathbf{U}}^{*}(\mathbf{U}, \psi)=j_{\mathbf{U}}, \quad \mathbf{V}(T)=0
$$

Therefore, with $\mathbf{V}$ solution of the backward adjoint equation (20) with the chosen final condition one has:

$$
\int_{(0, T) \times \Omega} j_{\mathbf{U}} \mathbf{U}_{\psi}=\int_{\Omega} \mathbf{V}(0) \mathbf{U}_{0}^{\prime}(\psi)-\int_{(0, T) \times \Omega} \mathbf{V} F_{\psi}(\mathbf{U}, \psi) .
$$


If there is no direct dependency between the initial condition $\mathbf{U}(0)$ and $\psi$ the first term in the right-hand-side vanishes. Also, for the Saint Venant equations the direct dependency in $\psi$ is in $g h \nabla \psi$ in the equations for $h \mathbf{u}$, see (7). Denoting $\mathbf{V}=\left(v_{1}, \mathbf{v}_{\mathbf{2}}\right)^{t}$ with $\mathbf{v}_{\mathbf{2}}$ the adjoint variable associated to $\mathbf{u}$, one has in weak form:

$$
\int_{(0, T) \times \Omega} \mathbf{V} F_{\psi}(\mathbf{U}, \psi)=-\int_{(0, T) \times \Omega} g \nabla \cdot\left(h \mathbf{v}_{\mathbf{2}}\right) .
$$

We have used either slip or Dirichlet boundary conditions for the velocity which give for the corresponding adjoint variable slip or homogeneous Dirichlet conditions removing the boundary term in weak form. An important point here is that, unlike with the linearized equation, with $\psi$ of any dimension $\mathbf{V}$ is computed only once. One remarks however that states $\mathbf{U}$ are needed in reverse order because the backward integration in $(20)$.

The previous analysis has been implemented by automatic differentiation [31, 7, 28] applied to a solver for the shallow water equations. In this code the equations are discretized by a finite volume formulation. Our finite volume implementation preserves steady state solutions on non flat sea beds in the absence of perturbations $[17,18]$. Four boundary conditions are needed at slip, inlet, shoreline and outlet boundaries. The slip boundary condition $(\mathbf{u} . \mathbf{n}=0)$ is naturally taken into account in a finite volume formulation. The outlet condition is a transmissive boundary condition. Values at boundary cells are obtained by second order extrapolations normal to the boundary from the values inside the domain. To describe incoming waves we use an absorbing/generating inlet boundary condition where the values of water depth are prescribed. In subcritical regimes, characteristic curves and Riemann invariants provide normal velocity. In open sea we allow for reflected waves to freely exit the domain [19]. Time integration is explicit for the flow. Due to what we said on the difference of time scales between fluid motion and changes in sea beds, several time steps will be taken (typically 100) in the flow solver before a new time step by the bed model described by the minimization iterations. In the examples in section 6 where flow comes from superposition of waves with a smallest 
period of 2.5s, 100 flow iterations imply at least 5 interactions between the fluid and the bed models in one period as the flow time step is around $0.005 \mathrm{~s}$ which gives a coupling time scale $T$ of $0.5 \mathrm{~s}$. Details on the solver are given in [29]. As this is not central in the paper we avoid extensive details on the solution procedures for the Saint Venant equations.

\section{Examples of shape adaptations following VaR-Based extreme SCENARIOS}

We consider a flat soft bed interacting with a flow governed by the shallow water equations. The way the bed responds to flow perturbations is defined in section 4. We would like to show the impact of the variability in the bed receptivity $\rho$ for which we assume Gaussian PDF around a constant value of $0.002 \mathrm{~m} / \mathrm{s}$ with $\operatorname{VaR}_{0.95}^{ \pm}(N(0, \sigma))= \pm f(x)$ linearly increasing cross-shore as in our simple model of section 3 and shown in figure 2 .

The initial bed is flat and waves are produced on the left boundary of the domain $\Omega$ representing a square wave basin de side $30 \mathrm{~m}$. To simulate sea conditions, the water wave elevation at the inlet is represented by the addition of $N$ monochromatic waves:

$$
h(t)=h_{0}+2 A \sum_{i=1, . ., N-1} \sin \left(\frac{\omega_{i}+\omega_{i+1}}{2} t\right) \cos \left(\frac{\omega_{i}-\omega_{i+1}}{2} t\right) .
$$

$A=0.12 \mathrm{~m}$ is the water wave amplitude at inlet, $h_{0}=0.765 \mathrm{~m}$ the water height at rest and $\omega_{i}=2 \pi / T_{i}$ are wave pulses. With two modes, typical values of periods $T_{i}$ we consider are $3.5 \mathrm{~s}$ and $2.5 \mathrm{~s}$. More realistic conditions can be applied following a Jonswap distribution $[24,23]$.

As specified in section 4.1, the functional we consider for this coupling aims at minimizing water elevations and their spatial gradients on a given time interval $T=0.5 s$. Figure 5 shows two scenarios for the bed after 400 minutes of interaction with the flow minimizing (11). The scenarios differ in the bed receptivity levels $(\rho=$ $0.0002 \mathrm{~m} / \mathrm{s}$ and $\rho=0.002 \mathrm{~m} / \mathrm{s}$ ). Three-dimensional structures appear in the higher receptivity case (with light sand) despite that the incoming wave is mono-directional. 
These disappear when the bed is made of materials with lower receptivity (with more heavy sand). The longshore variability of the bed shows the ratio between the large longshore structures and these 3D smaller ones. One sees that the global bed organization is faster in the high receptivity situation but there are small fluctuations in the time history of the functional corresponding to the oscillations of the small structures. These are better shown in the enlargement of the time history in figure 6. These oscillations do not exist in the heavy sand configuration. The reduction in the functional is visible comparing these histories to the evolution of the functional on a flat bed not adapting to the flow.

In the sequel, one only considers the higher receptivity setup (i.e. lighter sand with $\rho=0.002 \mathrm{~m} / \mathrm{s}$ ) and aims at discussing the variability issues analysis by the ingredients presented in the paper. Figure 6 shows the evolution of the local variability defined by $\sigma(t, x)$ and its evolution by equation (13). It also shows the time histories of the functional (11) without the variability accounted for and for the two extreme scenarios defined by $\operatorname{VaR}_{0.95}^{ \pm}(N(0, \sigma(t, x)))$.

Figure 7 shows the differences between the two final shapes and bed receptivity variabilities for the two extreme scenarios based on $\operatorname{VaR}_{0.95}^{ \pm}(N(0, \sigma(t, x))) \cdot \sigma(t, x)$ is governed by equation (13). One sees the importance of accounting for the uncertainty in the simulation and not only at the end through simple security margins as there is not necessary a simple spatial correlation between the shapes deviation and the spatial distribution of the uncertainty in the bed receptivity. Surprisingly, and unlike in the illustrative model problem of section 3, larger deviations between the two shapes do not take place where the bed receptivity features larger variability.

Figure 8 shows a snapshot of the bed velocity $V=\left(\rho \nabla_{\psi} / \partial_{x_{1}} \psi, \rho \nabla_{\psi} / \partial_{x_{2}} \psi\right)$. This field is evaluated by a postprocessing step from equation (12) and is never used in any calculation. The figure also shows the evolution in time of $\|V\|_{L^{2}(\Omega)}$. The shape never rests and continues to vary in time around structures organized in two different regions $(x \leqslant 20$ and $x>20)$. The shape is flat in average in the region $x>20$ as shown in figure 5 . 
Figure 9 illustrates the impact of the conservation constraint discussed in section 4.3 on the two extreme scenarios shown in figures 6 and 7 . One sees that, as the simulation time is long, one should pay attention to the fact that a closed basin is necessary different than an open sea domain. The deviation between the two beds increases with time. It is interesting that in the absence of the conservation constraint (i.e. open sea condition) the two scenarios predict opposite behaviors for the total sand volume over the domain. This is quite counter intuitive as one would have not expected such an impact from the variability on the bed receptivity. In addition, the total sand volume increases with the scenario using $\operatorname{VaR}_{0.95}^{-}(N(0, \sigma(t, x)))$ and decreases with $\operatorname{VaR}_{0.95}^{+}(N(0, \sigma(t, x)))$. This approach is therefore also useful to analyze the pertinence of an experimental setup.

\section{CONCLUDING REMARKS}

The Value at Risk concept has been introduced to define extreme scenarios for the motion of a soft bed under the action of a flow described by the Saint Venant shallow water equations. The approach, which enters the domain of shape optimization for unsteady fluids with one application being worst-case analysis in coastal erosion, is generic and applicable with other flow or bottom models. It permits to quantify our confidence on the bed evolution without any sampling of the bed characteristics parameter space, and one remains in the deterministic framework with supposed sufficient regularity where the gradient of the functionals can be defined. To go beyond the stationary case for the variance, the bed motion and its local variability have been linked through an original transport equation. It has been shown that one should account for the bed variability during the coupling between the fluid and bed motion models and not only eventually by the introduction of margins on the final results of a single deterministic simulation. A discussion on the impact of bed variability on sand conservation has shown a non intuitive impact of the former 
on the later. Also, an indicator has been provided to measure the pertinence of extending to open seas the conclusions of experiments performed in a basin.

Acknowledgments: The adjoint Saint Venant solver has been obtained using Tapenade AD tool developed at INRIA-Sophia Antipolis by L. Hascoet and his team Tropics.

\section{REFERENCES}

[1] Mandic, D. and Chambers, J. Recurrent Neural Networks for Prediction: Architectures, Learning Algorithms and Stability. John Wiley, London, 2001.

[2] Hoel, P.G. Introduction to Mathematical Statistics, John Wiley, London, 1971.

[3] Lindman, H.R. Analysis of Variance in Complex Experimental Designs, Freeman, New York, 1974.

[4] Spooner, J. T. Maggiore, M. Onez, R. O. and Passino, K. M. Stable Adaptive Control and Estimation for Nonlinear Systems: Neural and Fuzzy Approximator Techniques. John Wiley, New York, 2002.

[5] Veroy, K. and Patera, A. Certified real-time solution of the parametrized steady incompressible Navier-Stokes equations: Rigorous reduced-basis a posteriori error bounds Int. J. Numer. Meth. Fluids, 47(8), 773-788, 2005.

[6] Barré de Saint Venant A. J.-C. Théorie du mouvement non-permanent des eaux, avec application aux crues des rivières et à l'introduction des marèes dans leur lit. C.R. Acad. Sc. Paris; Vol. 73, 147-154, 1871.

[7] Mohammadi, B. Pironneau, O. Applied Shape Optimization for Fluids (2nd Edition), Oxford Univ. Press, 2009.

[8] Griewank, A. Computational derivatives, Springer, New York, 2001.

[9] Azerad, P. Bouchette, F. Isebe, D. Mohammadi, B. Optimal shape design of coastal structures minimizing short waves impact. Coastal Engineering. Vol. 55/1, 2008.

[10] Azerad, P. Bouchette, F. Isebe, D. Mohammadi, B. Shape optimization of geotextile tubes for sandy beach protection. International Journal for Numerical Methods in Engineering, Vol. 74, $1262-1277,2008$.

[11] Bouharguane A., Azerad P., Bouchette F., Marche F. and Mohammadi B., Low complexity shape optimization and a posteriori high fidelity validation, Disc. Cont. Dyn. Syst., Ser. B, 13, pp. 759-772, 2010. 
[12] Dean, R.G. Dalrymple, R.A. Coastal processes with Engineering Applications, Cambridge University Press, 2004.

[13] Dean, R.G. Dalrymple, R.A. Water Wave Mechanics for Engineers and Scientists, World Scientific Publishing, 1991.

[14] Mei, C.C. The applied dynamics of ocean surface waves. World Scientific Publishing, 1989.

[15] Dronkers, J. Dynamics of Coastal Systems, Adv. Ser. Ocean Eng. (25), World Scientific, Singapor, 2005.

[16] Nielsen, P., Coastal and Estuarine Processes. World Scientific, Singapore, 2002.

[17] Audusse, E. Bouchut, F. Bristeau, MO. Klein, R. Perthame, B. A fast and stable well-balanced scheme with hydrostatic reconstruction for shallow water flows. SIAM J.Sci.Comp. 25/6, 2050$2065,2004$.

[18] Audusse, E. Bristeau, M.-O. A well-balanced positivity preserving second order scheme for shallow water flows on unstructured meshes, J. Comp. Phys. Vol. 206, 311-333, 2005.

[19] Kobayashi. N. Desilva, GS. Watson, KD. Wave transformation and swash oscillation on gentle and steep slopes. J. Geophys. Res. Vol. 94, 951-966, 1989.

[20] Paola, C. Voller, V. R. A generalized Exner equation for sediment mass balance, J. Geophys. Res., 110, 2005.

[21] Yang, C.T. Sediment transport: theory and practice, Mc Graw Hill Education Europe, 1995.

[22] Fowler, A.C. Dunes and drumlins. Geomorphological fluid mechanics (eds. N.J. Balmforth and A. Provenzale), Springer-Verlag, Berlin, 430-454, 2001.

[23] Hasselmann, D.E. Dunckel, M. Ewing, J.A. (1980). Directional Wave Spectra Observed during JONSWAP 1973, Journal of Physical Oceanography, Vol. 10-8. 1264-1280.

[24] Hasselman et al. (1973). Measurements of Wind-Wave Growth and Swell Decay during the Joint North Sea Project (JONSWAP). Ergansungsheft, Reihe A(8), Nr. 12, Deutschen Hydrografischen Zeitschrift.

[25] Jorion Ph. Value at Risk: The New Benchmark for Managing Financial Risk, McGraw-Hill, 2006.

[26] Nocedal J., Wright S.Numerical Optimization, Springer, NY, 2006.

[27] Kapur K.C., Lamberson L.R.Reliability in Engineering Design, John Wiley \& Sons, New York, 1977.

[28] Mohammadi, B. Pironneau, O. Shape Optimization in Fluid Mechanics, Annual Revue of Fluid Mechanics, 36-1: 255-279, 2004.

[29] Bouharguane, A. Mohammadi, B. Optimal dynamics of soft shapes in shallow waters, Computers and Fluids, 40/1, 291-298, 2011. 
[30] Bouharguane, A. Mohammadi, B. Minimization principles for the evolution of a soft sea bed interacting with a shallow sea, IJCFD, 26/3, pp. 163-172, 2012.

[31] Hascoet, L. Pascual, V. Tapenade 2.1 user's guide. INRIA Technical Report RT-300, 2004.

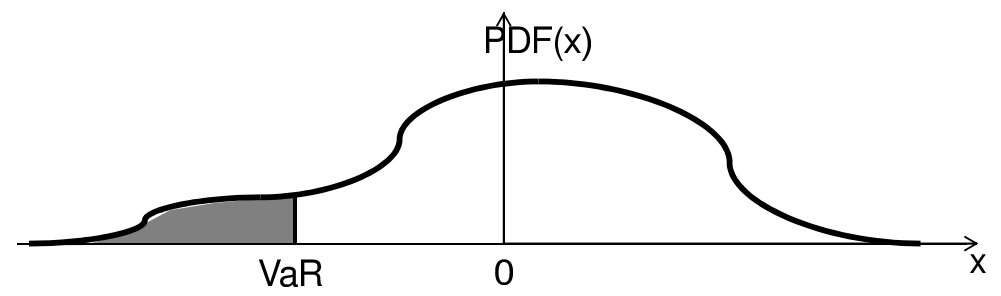

Figure 1. Value at risk for a general PDF. The grey area worths $\alpha$. 

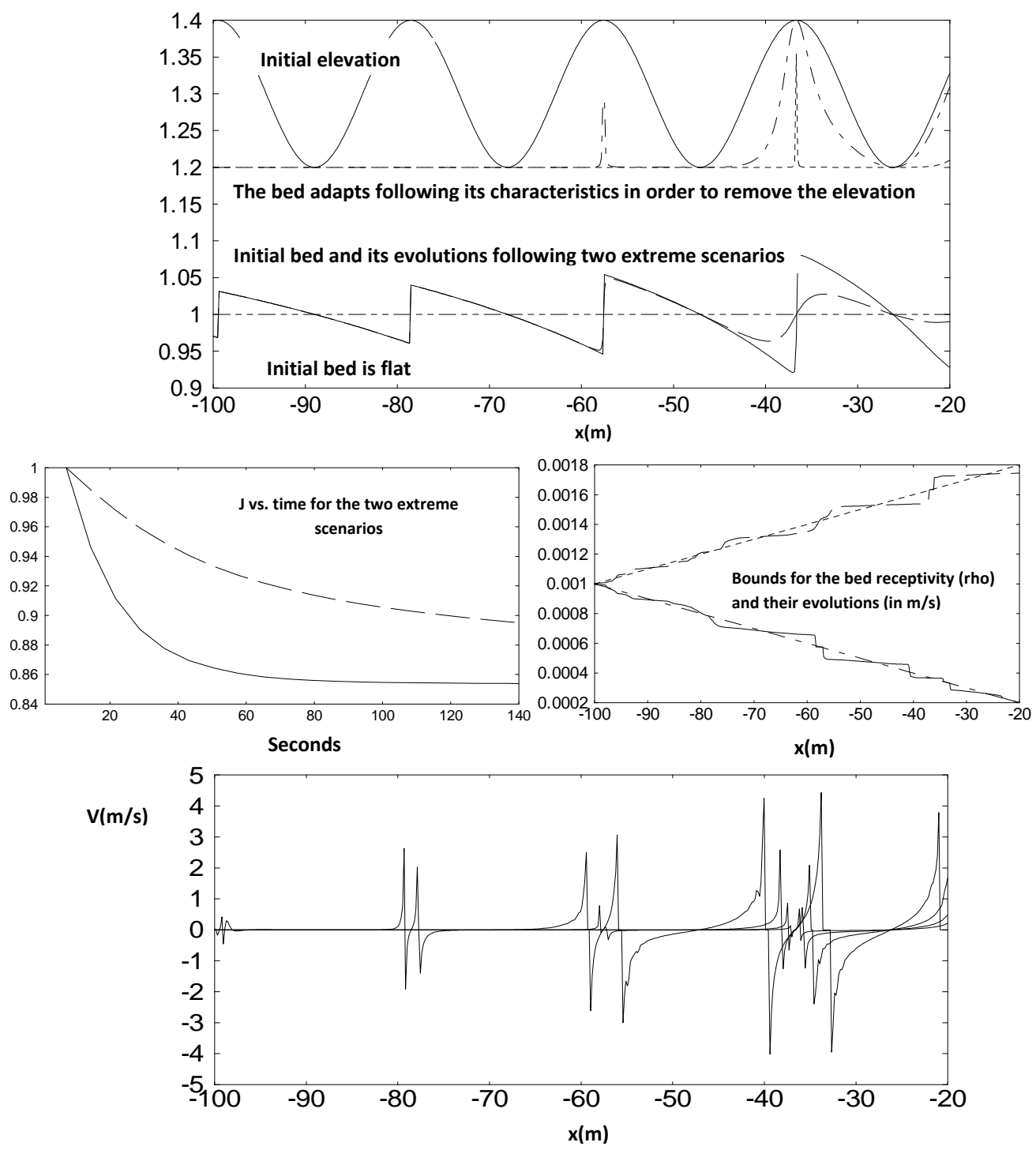

FiguRE 2. Upper: surface elevation $h$ and bed $\psi$ evolutions for the illustrative problem of section 3 subject to bed receptivity variability. Middle-left: reduction of the wave energy in time due to bed motion for the two extreme scenarios given by $\operatorname{VaR}_{0.95}^{ \pm}(N(0, \sigma))$. Middle-right: VaR based bounds for the bed receptivity and their evolution following 5. Lower: snapshots of $V(t, x)=\rho(t, x) \nabla_{\psi} J / \partial_{x} \psi$ the 'equivalent' speed of the motion of the bed during the coupling between the bed and elevation models (3). 

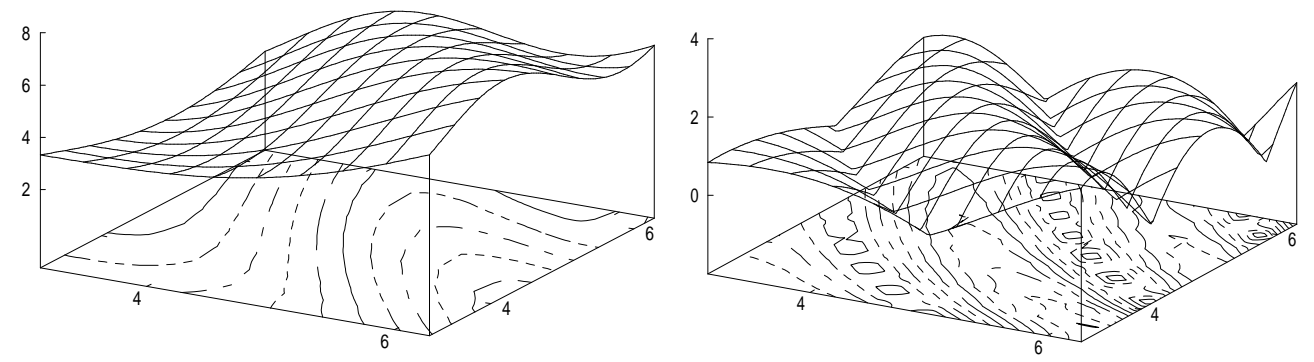

Figure 3. Example of bed $\psi$ and the inverse of the local time scale (right) in the solution of (13). When this vanishes, one should look for steady solutions making the problem stiff.
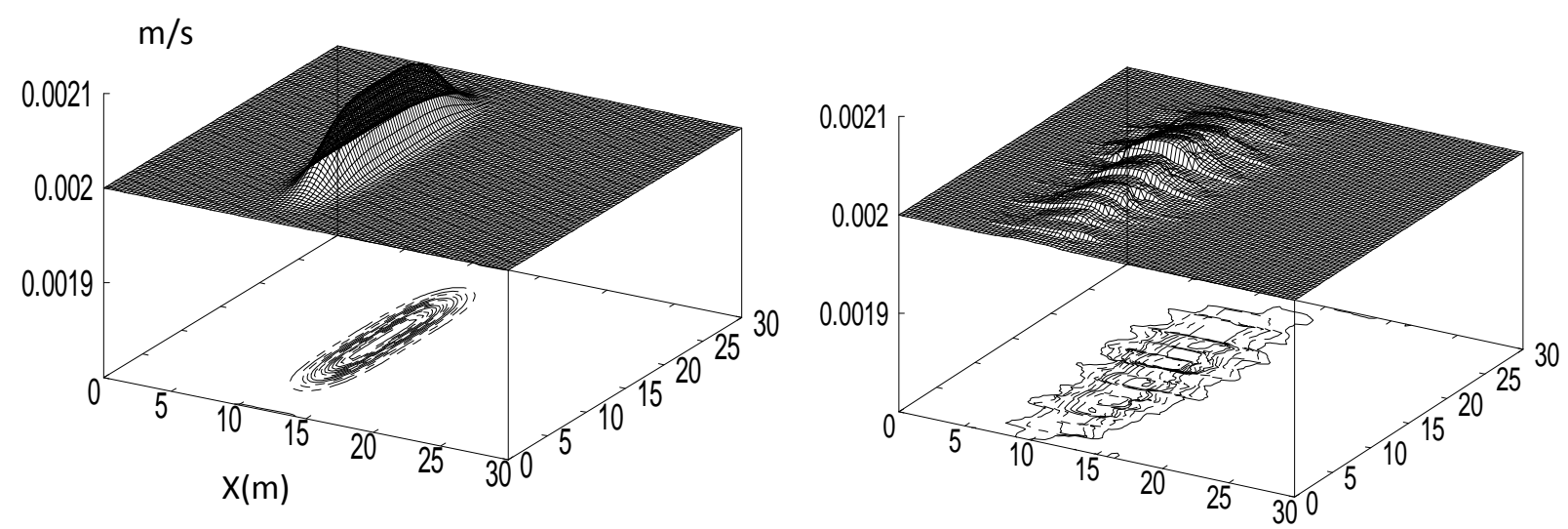

Figure 4. Example of a locally perturbed initial constant bed receptivity (left) and its dispersion governed by equation (13) (right). 


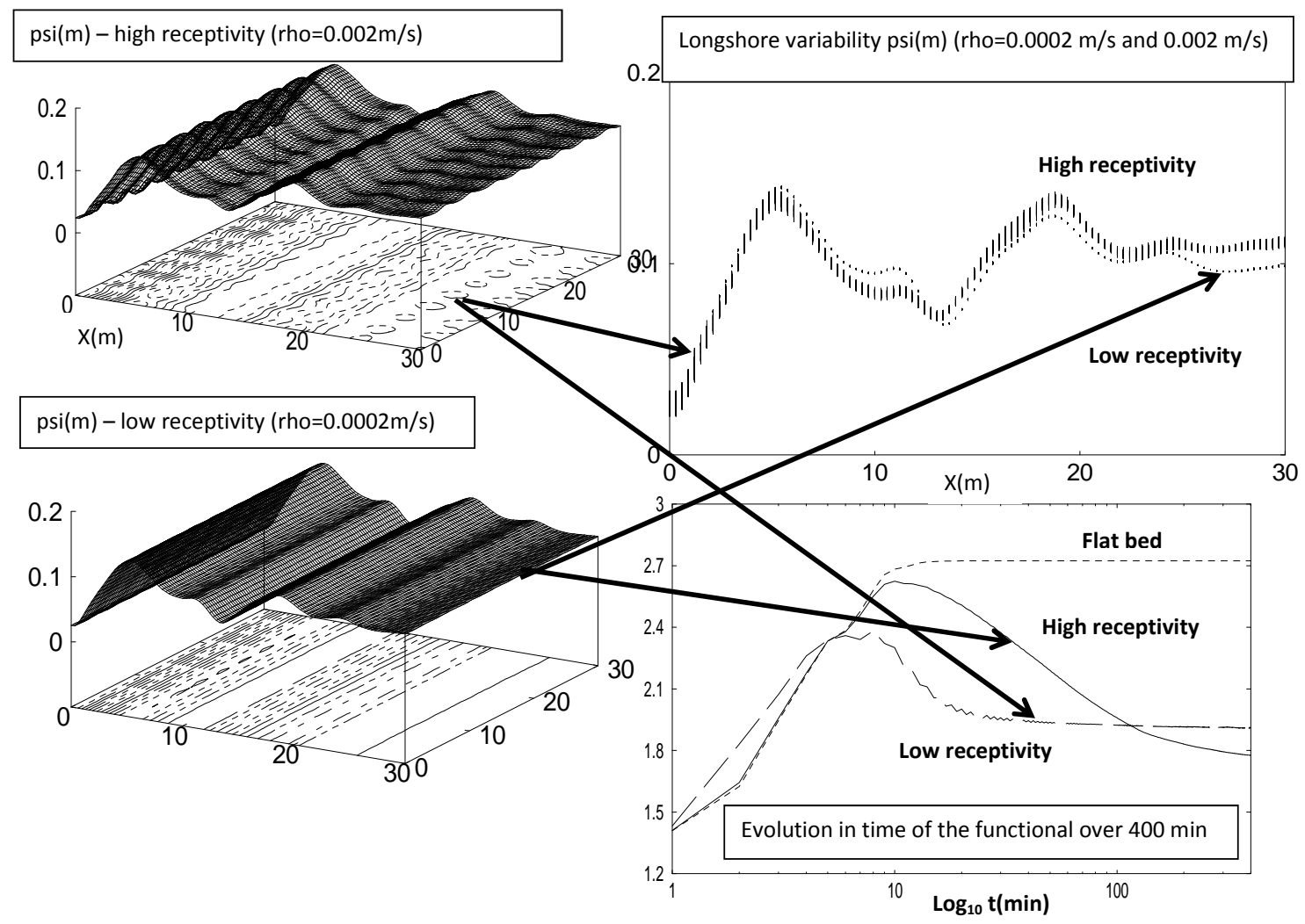

Figure 5. The final shapes (left) for two levels of bed receptivities after 400 minutes of interaction between the bed and the flow and their longshore variabilities (top-right). The time evolutions of the functional for the two setups are compared to the situation where the bed is kept flat and does not adapt to the flow. 

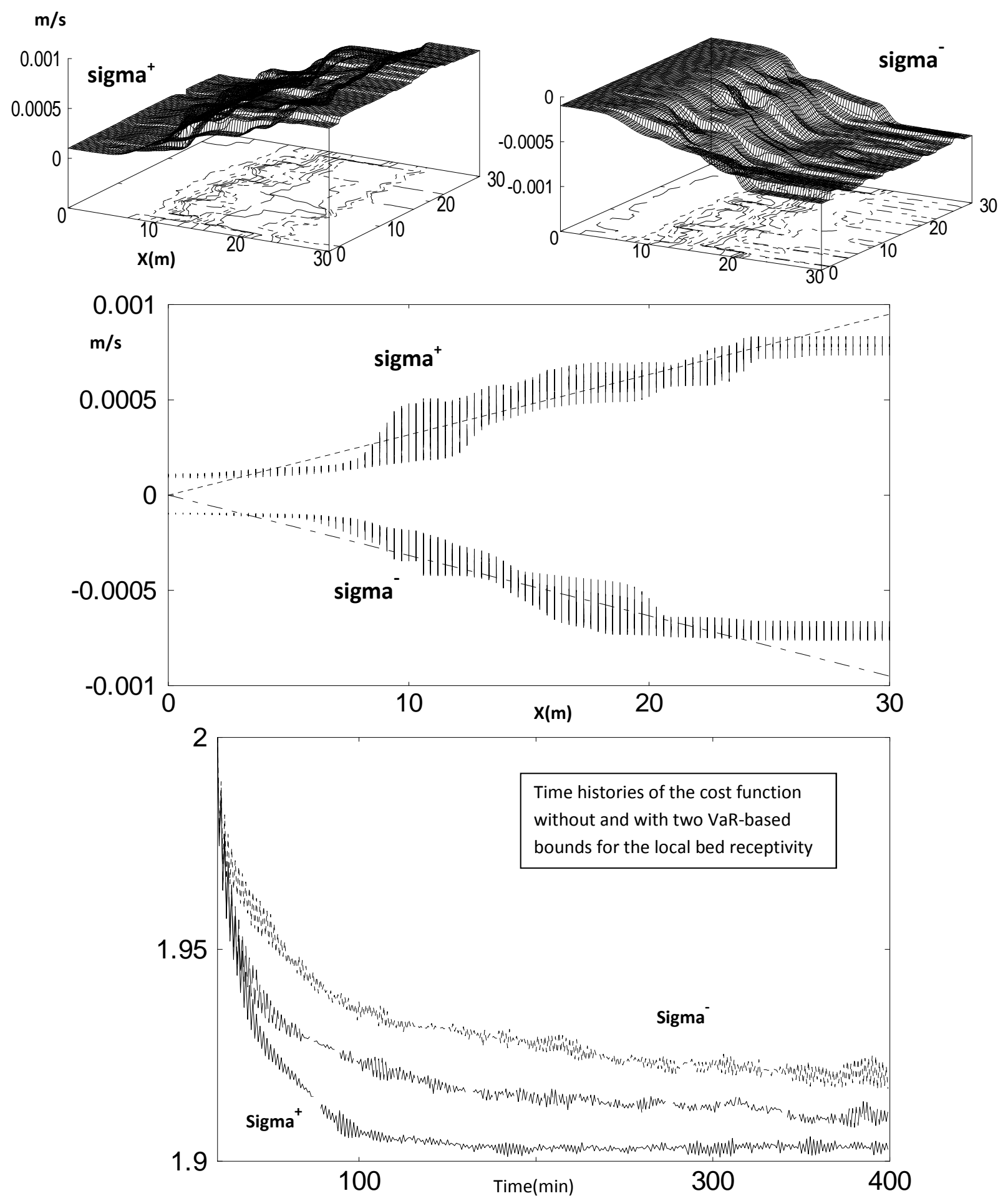

FiguRE $6 . \sigma^{ \pm}$corresponding to the two extreme scenarios defined by $\operatorname{VaR}_{0.95}^{ \pm}(N(0, \sigma(t, x))) \cdot \sigma^{ \pm}$initialisation are linear cross-shore through the domain as shown and their dispersions governed by equation (13). Lower picture is the time histories of the functional (11) without variability and with two VaR-based scenarios for the local bed receptivity $\rho(x, t)$. 


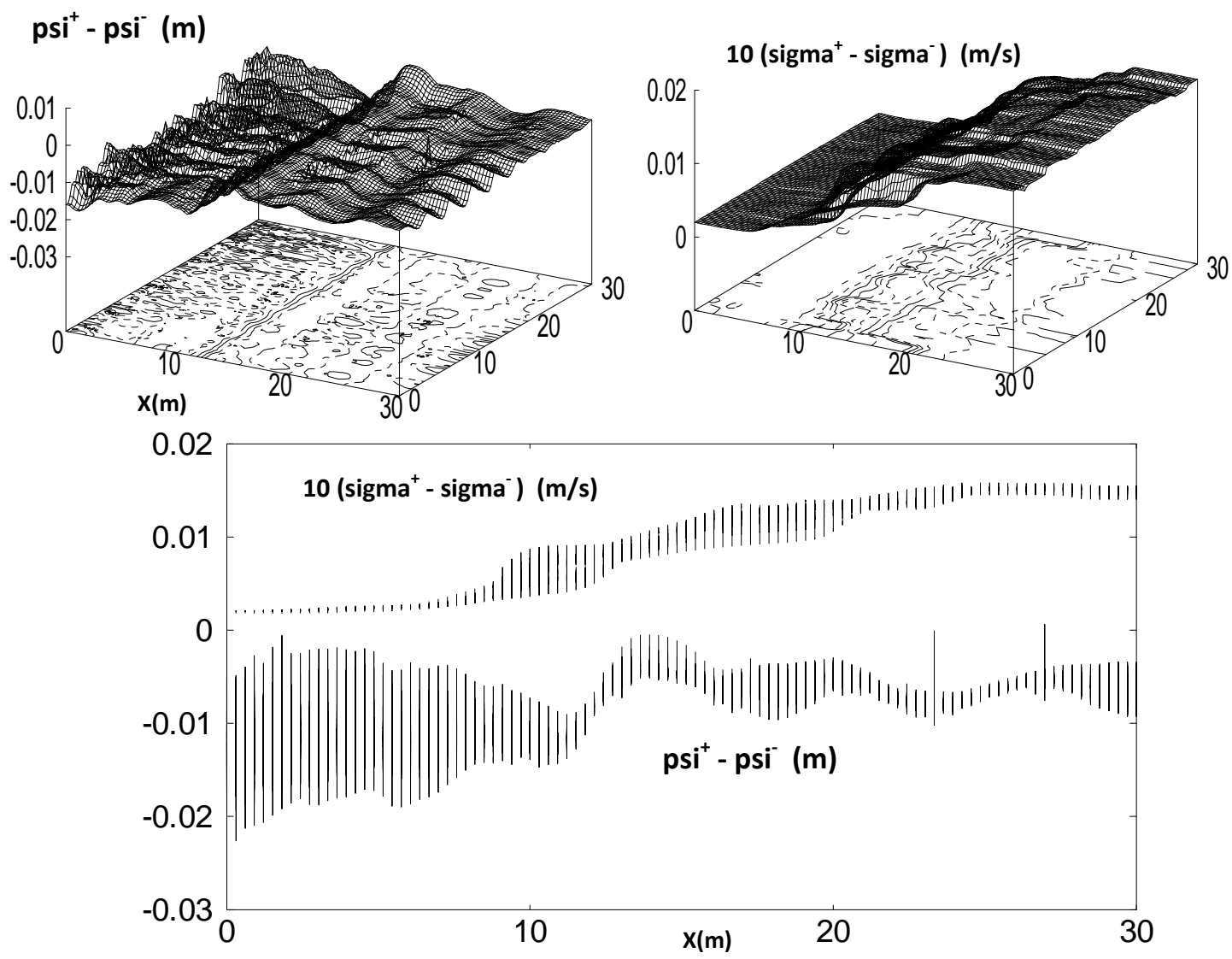

Figure 7. Top left: deviation between the two final shapes reached accounting for the two extreme scenarios for the bed receptivity. Top right: deviation between $\sigma^{ \pm}$corresponding to the two extreme scenarios defined by $\operatorname{VaR}_{0.95}^{ \pm}(N(0, \sigma(t, x)))$. Lower pictures is the longshore variabilities of these showing that larger deviations do not necessarily appear where variability in bed receptivity is large. 


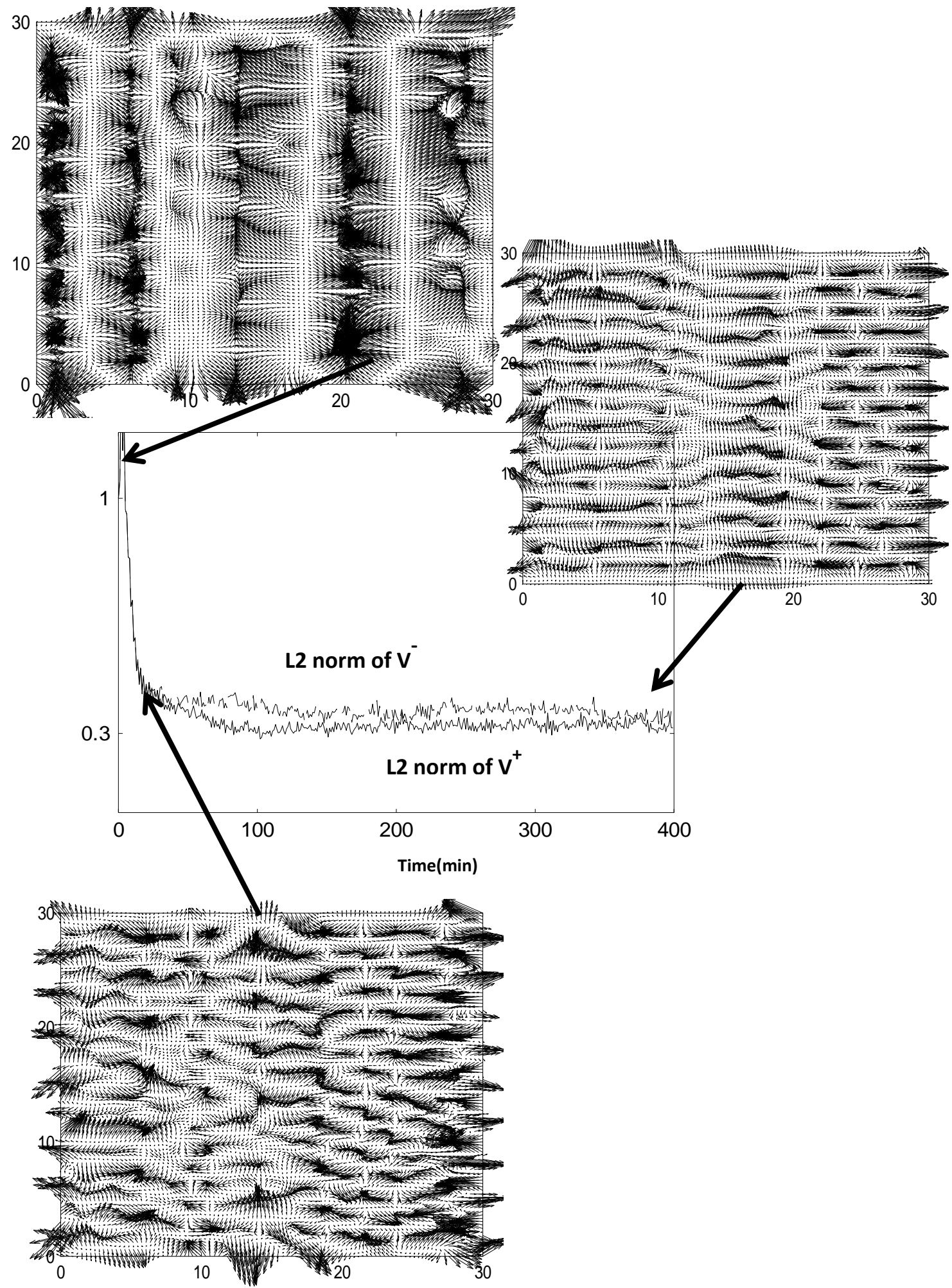

Figure 8. Snapshots of $V$ at three different stages as indicated and the time histories of the $L^{2}$ norm of $V$ for the two extreme scenarios defined by $\operatorname{VaR}_{0.95}^{ \pm}(N(0, \sigma(t, x)))$. The outcome is not intuive as the fluctuations in the simulation with the lower bound $\mathrm{VaR}_{0.95}^{-}$in variability remain larger than with $\mathrm{VaR}_{0.95}^{+}$. 

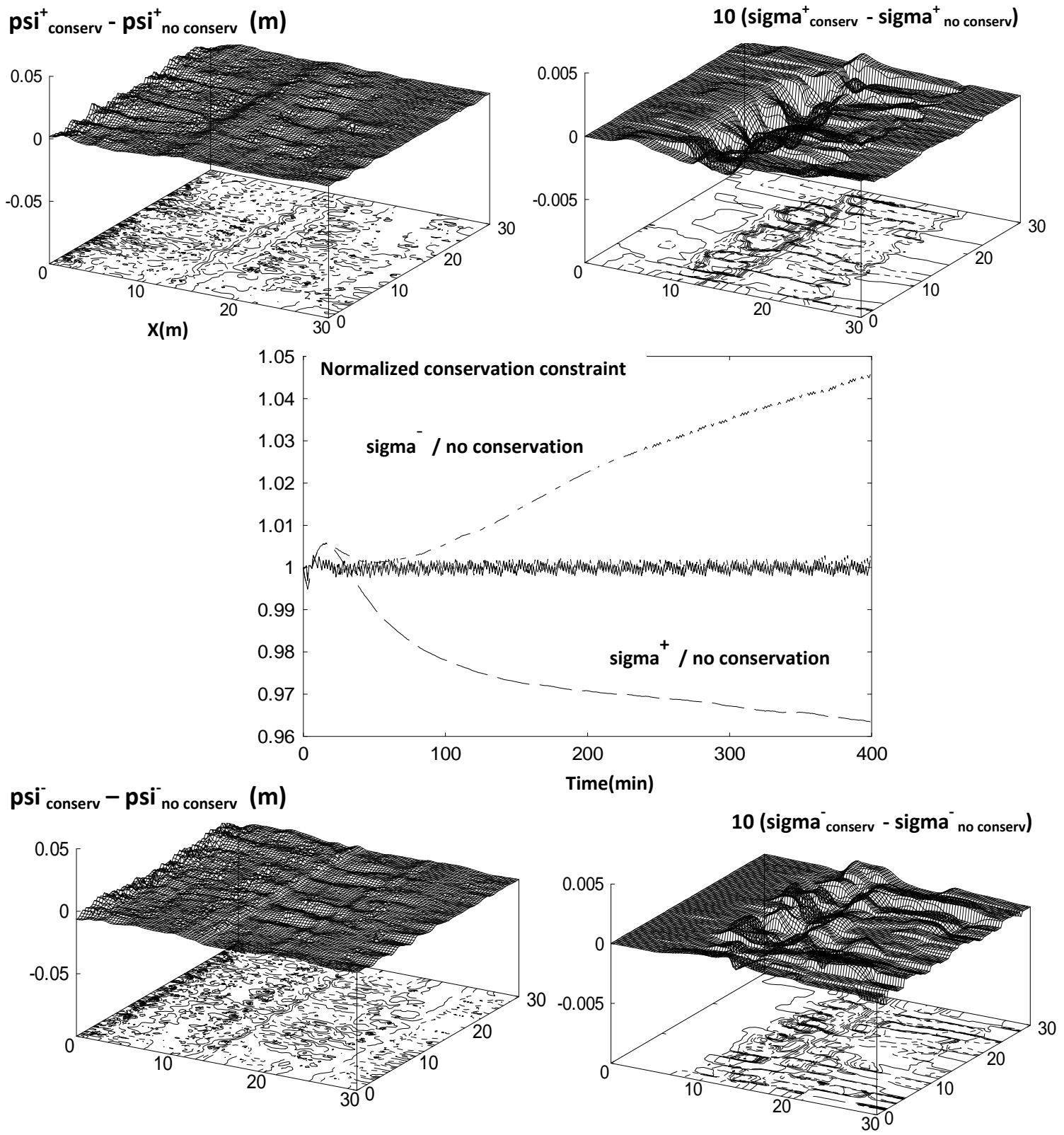

Figure 9. Top left: deviation between the two final shapes reached for the extreme scenarios by $\operatorname{VaR}_{0.95}^{+}(N(0, \sigma(t, x)))$ with and without the conservation constraint enforced and on the top right the deviation between the corresponding $\sigma^{+}$(same in bottom left and right but with $\left.\operatorname{VaR}_{0.95}^{-}(N(0, \sigma(t, x)))\right)$. Middle picture is the evolution in time of the normalized conservation constraint for these four scenarios. 